THE GEM OBI/IC443/S249 COMPLEX: A CASE HISTORY OF STELLAR EVOLUTION

\author{
R. Braun \\ Leiden Observatory, The Netherlands \\ R.G. Strom \\ Dwingeloo Observatory, The Netherlands
}

The extended cloud complex containing members of the Gem OB1 association, the supernova remnant IC443, and the HII region S249 has been studied with IRAS observations at $12,25,60$ and 100 microns and WSRT observations at 327 and $1400 \mathrm{MHz}$ and in the $21-\mathrm{cm} \mathrm{HI}$ line. A skeleton-1ike framework of cool dust delineates the boundaries of the region, and physical parameters have been derived for the entire complex, individual $H$ II regions and the shocked and recombined gas within IC443 using the radio and infrared data. IC443 is shown to consist of three interconnected, roughly spherical subshells of vastly different radii and centroids. The geometry is fully constrained by the structural and kinematic data. Two of the subshells together define the usually assumed boundaries of IC443, while the third includes the optical filaments which extend beyond the northeastern rim and which are shown to have we11-correlated nonthermal radio components. The available evidence implies that the SNR shock has encountered a pre-existing high density shell. It is shown that the system of subshells is fully consistent with formation by stellar wind driven bubbles generated by association members within the inhomogeneous environment of the complex.

\title{
A NEW THEORY OF STAR FORMATION: ANALYTICAL MASS FUNCTION AND STAR
} FORMATION RATE

\author{
A. Di Fazio \\ Osservatorio Astronomico di Roma \\ Viale del Parco Mellini 84, I-00136, Roma, Italy
}

An analytical, theoretical, time-dependent initial mass function is derived for the objects created in the fragmentation of a gravitationally unstable gas protocloud. The mass spectrum depends on the chemicaldynamical-radiative evolution of the protocloud and it peaks at a mass slightly greater than the minimum Jeans mass attained throughout the evolution. A fragmentation rate mass spectrum is also analytically derived.

By using an evolutionary model presented here, it is shown that the fragmentation process implies the existence of several generations of 
fragments, with different ranges of mass. A proto-galactic evolution model, using our new theory, predicts that the first stars are created in the third generation of fragments. These can represent the "zerometal" population.

Mass functions for various objects are elaborated from observational data. Their shapes are compared among themselves quantitatively. A striking similarity suggests the hypothesis that all the different kinds of astrophysical objects examined were formed by essentially the same process. The theoretical mass functions predicted by the evolutionary models and the fragmentation rate are quantitatively compared with the observations, yielding a remarkably good agreement.

STRUCTURE AND FRAGMENTATION OF MOLECULAR COMPLEXES

E. Falgarone $e^{1,2}$, J.L. Puget ${ }^{1,2}$, M. Pérault ${ }^{1,2}$,

S. Bonazzola ${ }^{2}$, J. Heywaerts ${ }^{2}$

${ }^{1}$ Radioastronomie, Laboratoire de Physique, ENS, 24 rue Lhomond, 75005 Paris, France

${ }^{2}$ Observatoire de Paris, 92190 Meudon, France

We propose a hierarchical model of large molecular complexes (mean density $\sim 10 \mathrm{~cm}^{-3}$, masses larger than $10^{5} \mathrm{M}_{\odot}$ relevant to their "cold phase") which preceeds the formation of massive stars and H II regions, and investigate the gravitational stability of the different scales.

The parameters of the hierarchy are mostly derived from observations: its building blocks are clouds of well defined characteristics (mean density $200 \mathrm{~cm}^{-3}$ masses $\sim$ a few hundreds solar masses), virialized in the potential well of the complex. They may form subgroups of all sizes up to that of the largest scale.

The volume filling factor of the clouds in the parent complex is low $\left(f_{v} \sim 10^{-2}\right)$, the bulk of its volume being filled with a low density gas: the warm HI phase.

First, we propose and investigate an efficient mechanism to transfer kinetic energy from the orbital motions of the clouds to their internal random motions. The large perturbations of the magnetic field induced, at the clouds boundaries, by their interactions with their neighbours, excite systems of hydromagnetic waves trapped inside the clouds. The internal velocity dispersion of the clouds computed in this way is comparable to the values deduced from observed linewidths.

The pumping rate allows the cloud internal velocity dispersion which has a short hydrodynamic timescale $\left(\tau=2 \mathrm{R} / \mathrm{V} \sim 3 \times 10^{6} \mathrm{yr}\right)$ to be fed over more than several $10^{7} \mathrm{yr}$. In addition the energy supply is such that in a cloud contraction its internal energy increases at fast as the gravitational potential energy: the clouds are therefore stable against radius perturbations. 